role in the study design, data collection and analysis, decision to publish, or preparation of the manuscript. The authors declare that they have no competing interests.

\section{PRELIMINARY RESULTS OF ANLOTINIB AND NIRAPARIB DUAL THERAPY EVALUATION IN PLATINUM-RESISTANT RECURRENT OVARIAN CANCER (ANNIE): A MULTICENTER, SINGLE-ARM, PHASE 2 TRIAL}

${ }^{1}$ Guochen Liu, ${ }^{1}$ Jihong Liu, ${ }^{2}$ Bingna Xian, ${ }^{3}$ Yanling Feng, ${ }^{3}$ Qidan Huang. ${ }^{1}$ Sun Yat-Sen University Cancer Center; Department of Gynecologic Oncology; ${ }^{2}$ Sun Yat-Sen University Cancer Center; ${ }^{3}$ Department of Gynecologic Oncology

\subsection{6/ijgc-2020-ESG0.105}

Introduction/Background Patients with platinum-resistant ovarian cancer have a poor prognosis. Effective treatment options for these patients are limited. In this study (ANNIE), we evaluate the activity of niraparib combined with anlotinib in patients with platinum resistant recurrent ovarian carcinoma.

Methodology The ANNIE trial was a multicentre, single-arm, phase 2 study that evaluated the safety and activity of niraparib combined with anlotinib in patients $(\geq 18 \& \leq 70$ years) with recurrent ovarian epithelial, fallopian tube, or primary peritoneal cancer cancer whose disease recurred in less than 6 months after the last administered platinum therapy. Patients received oral niraparib $300 \mathrm{mg} / 200 \mathrm{mg}$ once daily continuously and anlotinib 12 mg on day 1-14 of each 21-day cycle thereafter until disease progression or intolerable toxicity. The primary objective was to assess objective response rate (ORR; complete plus partial responses). 40 cases are planned to be enrolled.

Results Between May 22, 2020 and August 3, 2020, we enrolled 9 patients (median age, 54 years [range, 44-64 years]). Patients had received a median of five (range, 2-8) previous lines of therapy. All but one (voluntarily withdrew) of the patients were still on treatment, the longest has been taking medication for more than 4 cycles. Three patients underwent imaging evaluation, including 1 confirmed complete responses, 1 with confirmed partial responses, 1 with stable disease. No drug-related grade 3 or worse treatment-emergent adverse events were detected, the most common treatment emergent adverse events were hypertension (5 of 9 patients), hand-foot skin reaction (4 of 9 patients), hoarseness (4 of 9 patients). Enrollment was ongoing so far.

Conclusion It seems niraparib in combination with anlotinib is tolerable, with promising antitumor activity for patients with platinum resistant recurrent ovarian cancer. Besides, we observed unusual safety signals in the combination (more hoarseness and less haematological toxicities). The conclusion can be clarified after the research is completed.

Disclosures Trial registration ClinicalTrials. gov identifier: NCT04376073, Funding: Zai Lab, The authors declare no conflicts of interest.

\section{\begin{tabular}{l|l}
247 & OPTIMISING PREDICTION ACCURACY OF COMPLETE
\end{tabular} CYTOREDUCTION FOR HIGH GRADE SEROUS ADVANCED OVARIAN CANCER PATIENTS USING NEAREST-NEIGHBOR MODELS}

Alexandros Laios, Yong Tan, Richard Hutson, George Theophilou, Diederick Dejong. St James's University Hospital; Leeds Teaching Hospitals; Gynaecologic Oncology
Introduction/Background The foundation of modern ovarian cancer care is cytoreductive surgery to remove all macroscopic disease (R0). Identification of R0 resection patients may help individualise treatment. Machine learning and AI have been shown to be effective systems for classification and prediction. For a disease as heterogenous as ovarian cancer, they could potentially outperform conventional predictive algorithms for routine clinical use. We investigated the performance of an $\mathrm{AI}$ system, the k-nearest neighbor (k-NN) classifier to predict R0, comparing it with logistic regression.

Methodolog A cohort of patients diagnosed with high grade serous advanced ovarian, tubal and primary peritoneal cancer (HGSOC), undergoing surgical cytoreduction from 20152019, was selected from the ovarian database. Performance variables included age, BMI, Charlson Comorbidity Index (CCI), timing of surgery, surgical complexity and disease scores. The k-NN algorithm classified R0 vs non-R0 patients using 3-20 nearest neighbors. Prediction accuracy was estimated as percentage of observations in the training set correctly classified. The relative importance of the selected variables was quantified by calculating the prediction accuracy/ error rate in relation to the number of predictors included in the models.

Results 154 patients were identified, with mean age of $64.4+$ 10.5 yrs, BMI of $27.2+5.8$ and mean SCS of $3+1$ (1-8). Complete and optimal cytoreduction was achieved in $62 \%$ and $88 \%$ patients. The mean predictive accuracy was $66 \%$ compared to $63.4 \%$ by logistic regression. R0 resection prediction of true negatives was as high as $90 \%$ using $\mathrm{k}=20$ neighbors. From the variables tested to contribute in R0 prediction, only disease score was statistically significant $(\mathrm{p}=0.0006)$. For a given neighborhood size $\mathrm{k}=15$, $\mathrm{R} 0$ resection was best

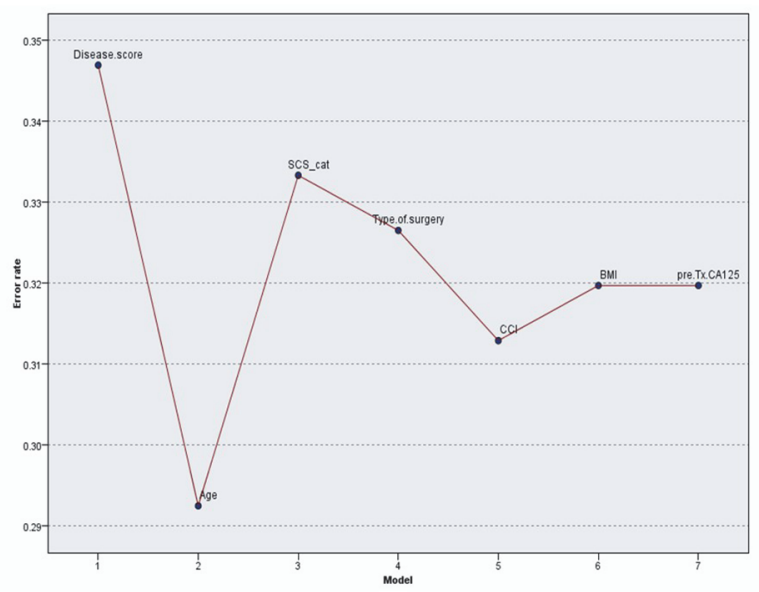

Abstract 247 Figure 1

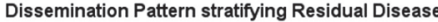

R1 R0

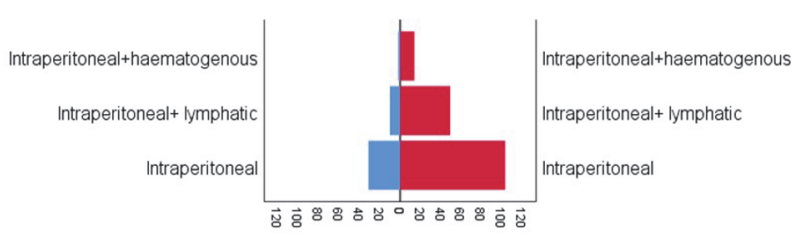

Abstract 250 Figure 1 
predicted by a $\mathrm{kNN}$ model that included age and CCI (figure 1).

Conclusion The k-NN algorithm is a versatile and promising tool for R0 resection in HGSOC patients, which outperforms logistic regression. The model, which is very much reflective of 'previous clinical experience' can be directly available to clinicians and is expected to improve accuracy with data expansion.

Disclosures No disclosures.

\section{SURVIVAL IMPLICATION OF PRE-TREATMENT IMAGING TUMOR DISSEMINATION PATTERN IN PATIENTS SURGICALLY TREATED FOR ADVANCED HIGH GRADE SEROUS OVARIAN CANCER}

Alexandros Laios, Yong Tan, Angelika Kaufmann, Mohamed Otify, Richard Hutson, Amudha Thangavelu, George Theophilou, David Nugent, Diederick Dejong. St James's University Hospital; Leeds Teaching Hospitals; Gynaecologic Oncology

\subsection{6/ijgc-2020-ESGO.107}

Introduction/Background Clarity and precision about the anatomical extent of disease in cancer is essential for prognostication, research, and cancer-control activities. To select effective therapeutic approaches for advanced high-grade serous ovarian cancer (HGSOC), yet the most prevalent and lethal form, it is important to identify stratification factors that could accurately predict prognosis before initial intervention. We hypothesized that women with different tumor dissemination patterns at pre-treatment imaging would have different prognosis.

Methodology This was a retrospective analysis of 209 FIGO stage III-IV HGSOC women, who were scheduled for cytoreductive surgery in SJUH Leeds between Jan 2015 to Dec 2018 with curative or life-prolonging intent. CT scans were reported by an MDT radiologist. Three pre-treatment imaging dissemination patterns were identified and verified by final histology. A Cox proportional hazard analysis was used to test the effect of imaging dissemination patterns, age, performance status (PS), timing of surgery (upfront vs delayed cytoreduction), surgical complexity score (SCS), residual disease (RD), disease score, and type of chemotherapy on survival. KaplanMeier survival curves were produced using SPSS ${ }^{\circledR} 26$.

Results There were no statistical differences in the cytoreduction rates amongst the three groups (figure 1). The mean progression free survival (PFS) for patients grouped as intraperitoneal $(n=137)$, intraperitoneal and lymphatic $(n=56)$, and intraperitoneal and haematogenous $(\mathrm{n}=16)$ was 26.5 (95\% CI 23.4-29.6), 21.3 (95\% CI 18.3-24.4) and 19.1 months (95\% CI 15.1-22.9), respectively. The mean overall survival (OS) was 45.8 (95\% CI 41.5-50.2), 34.8 (95\% CI
29.2-40.3) and 30.7 months (95\% CI 24.5-36.9), respectively $(p=0.05)$ (figure 2). The mean PFS and OS for the entire cohort was 25 months (95\% CI 22.6-27.3) and 41.8 (95\% CI 38.3-45.2), respectively. For PFS, Cox regression analysis identified PS (HR 1.23, 95\% CI 1.1-1.5, p=0.04), RD (HR $0.69,95 \%$ CI $0.46-0.98, \mathrm{p}=0.05)$ as statistically significant. For OS, Cox regression analysis identified PS (HR 1.47, 95\% CI 1.14-1.89, $\mathrm{p}=0.03$ ), dissemination pattern (HR 1.36, 95\% CI $1.02-1.86, p=0.05$ ) as statistically significant.

Conclusion For HGSOC prognosis, one should consider not only the patient's disease burden but also their overall medical status and ability to undergo extensive surgery. Prolonged survival rates were found predominantly in those patients with intraperitoneal only pre-treatment imaging dissemination pattern. Baseline tumor dissemination pattern can be a prognostic factor for overall survival. Classification of such patterns can help counsel patients initially on their prognosis and identify those who might benefit from intraperitoneal chemotherapy.

Disclosures No disclosures.

\section{CONTRASTING CLINICAL CHARACTERISTICS AND TREATMENT PATTERNS IN WOMEN WITH NEWLY DIAGNOSED ADVANCED-STAGE OVARIAN CANCER IN AUSTRALIA, SOUTH KOREA AND TAIWAN}

${ }^{1}$ Hung-Hsueh Chou, ${ }^{2}$ Anna Defazio, ${ }^{3}$ Byoung-Gie Kim, ${ }^{4}$ Chih-Long Chang, ${ }^{5}$ David Bowtell, ${ }^{6}$ Heng-Cheng Hsu, ${ }^{5}$ Nadia Traficante, ${ }^{3}$ Soo Young Jeong, ${ }^{7}$ Wen-Fang Cheng, ${ }^{5}$ Sian Fereday. ${ }^{1}$ Cgmh-Lk Chang Gung Medical Foundation, Linkou Branch; ${ }^{2}$ Centre for Cancer Research, The Westmead Institute for Medical Research; The University of Sydney; Department of Gynaecological Oncology, Westmead Hospital; ${ }^{3}$ Samsung Medical Center, Sungkyunkwan University School of Medicine; ${ }^{4}$ Mackay Memorial Hospital; ${ }^{5}$ Peter Maccallum Cancer Centre; Sir Peter Maccallum Cancer Centre Department of Oncology, The University of Melbourne; ${ }^{6}$ National Taiwan University Hospital, Hsin-Chu Branch; ${ }^{7}$ National Taiwan University Hospital

\subsection{6/ijgc-2020-ESG0.108}

Introduction/Background Epithelial ovarian cancer (EOC) is often associated with diagnosis at an advanced stage, poor prognosis and high mortality. Limited data exist on the clinical management of ovarian cancer (OC) patients in the AsiaPacific region. We evaluated secondary databases from Australia, South Korea and Taiwan to review the current standard of care in a real world setting prior to the introduction of poly-(adenosine diphosphate-ribose) polymerase inhibitor (PARPi) maintenance after first-line chemotherapy.

Methodology Data from medical records of nearly 1,000 women diagnosed with advanced-stage EOC in a 5 year period, between January 2014 and December 2018 were obtained from clinic- (Taiwan, South Korea) and cohort-based
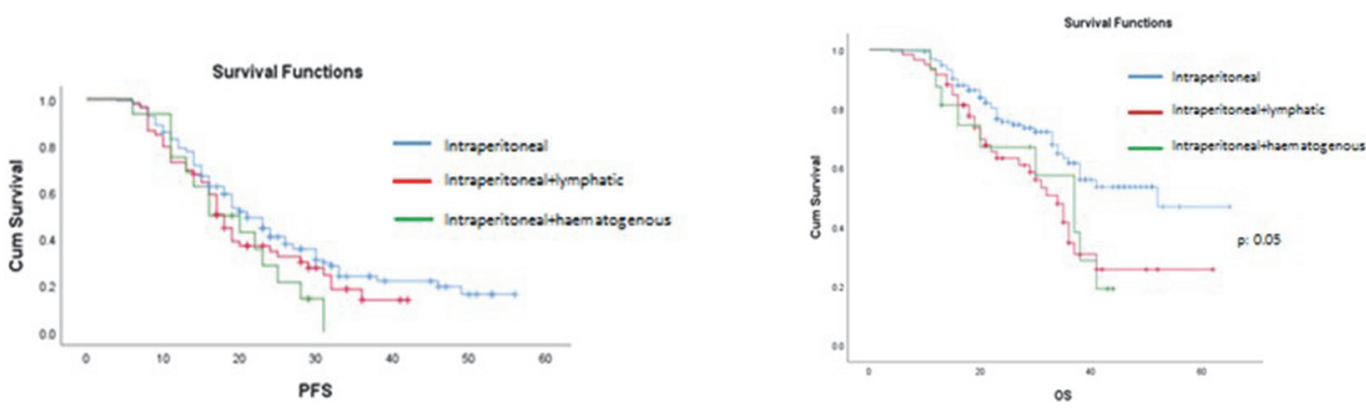

Abstract 250 Figure 2 